Revista de Filología Alemana

ISSN: 1133-0406

\title{
El uso de Volk en los programas de los partidos políticos alemanes y austríacos para las Elecciones Generales de 2017: un estudio contrastivo ${ }^{1}$
}

\author{
Miguel Ayerbe Linares ${ }^{2}$
}

Recibido: 24 de mayo de 2018 / Aceptado: 20 de julio de 2018

Resumen. Al revisar los programas electorales de los partidos que presentaron candidaturas en las últimas elecciones generales de 2017 en Alemania y Austria, llama la atención que el uso de VOLK no se limita únicamente a los partidos populistas. No obstante, se plantea la pregunta acerca de si VOLK significa siempre lo mismo, tanto semántica como referencialmente, con independencia de quien lo use. Más exactamente, ¿quién estaría incluido y quién excluido al usar VOLK? Partiendo de un análisis léxico de tipo cuantitativo y cualitativo de corpus, el presente trabajo se centra en el estudio de esta cuestión, analizando los programas electorales de los partidos que, en ambos países, se presentaron a las mencionadas elecciones.

Palabras clave: Análisis del discurso; Volk; Alemania; Austria; programas electorales.

\section{[en] The Use of Volk in German and Austrian Manifestos for 2017 General Elections: a Contrastive Analysis.}

\begin{abstract}
A scrutiny of the electoral manifestos of the different parties presenting candidacy for the past 2017 general elections in Germany and Austria reveals that the use of VOLK is not limited to populist parties. At this point the question arises whether VOLK always means the same either semantically or referentially, regardless of political party. More specifically, who is included and who is excluded in the term VOLK? By means of a quantitative and qualitative lexical analysis this paper focuses on this matter analyzing the manifestos of the parties that took part in the general elections of both countries.
\end{abstract}

Keywords: Discourse Analysis; Volk; Germany; Austria; Manifestos.

Sumario. 1. Introducción y objetivo. 2. Metodología. 3. Corpus de VOLK en los programas electorales de 2017 y su análisis. 3.1. VOLK en programas electorales alemanes. 3.2. VOLK en programas electorales austríacos. 4. Discusión en torno del uso de VOLK en Alemania y Austria. 5. Posible marco para el uso u omisión de VOLK por parte de los partidos políticos. 6. Conclusión

Cómo citar: Ayerbe Linares, M., «El uso de Volk en los programas de los partidos políticos alemanes y austríacos para las Elecciones Generales de 2017: un estudio contrastivo», Revista de Filología Alemana 27 (2019), 177-196

\footnotetext{
1 Trabajo realizado en el marco del proyecto de investigación FFI2015-65252-R "Imagining the People in the new Politics", financiado por el Ministerio de Economía y Competitividad.

2 Universidad del País Vasco / Euskal Herriko Unibertsitatea (España)

E-mail: miguel.ayerbe@ehu.eus
} 


\section{Introducción y objetivo}

En un año, el 2017, fueron convocadas elecciones generales en Alemania y Austria. Podría haberse tratado de una convocatoria más, si no hubiera sido por un aspecto que provocó una elevada expectación en ambos acontecimientos: de un lado, la concurrencia de Alternative für Deutschland (AfD), en Alemania, y, de otro, el Freiheitliche Partei Österreichs (FPÖ), en Austria. Ambos partidos son tildados de "populistas (radicales) de derechas", y, concretamente en Alemania, la formación de AfD había desatado de forma especial en los últimos meses una gran expectación a raíz de su posible entrada en el Bundestag, hecho que a día de hoy ya es una realidad.

A ambas formaciones políticas se les reprocha con frecuencia una actitud xenófoba ante la inmigración, especialmente a raíz de las recientes olas migratorias, así como de azuzar miedos frente a supuestas amenazas de invasiones encubiertas por parte de la inmigración, que estarían poniendo en peligro las identidades alemana y austríaca. Tampoco son desconocidas las polémicas surgidas en torno a declaraciones en boca de algunos de sus militantes, referidas a las cuestiones antes mencionadas o a expresiones concretas. Y es que el lenguaje juega un papel muy importante en el discurso político, según la perspectiva desde la que se mire: los diferentes partidos políticos, por un lado, buscan aquellos términos y formulaciones que mejor representen su propia postura (stance) e identidad, ya sea en el nivel denotativo como connotativo; los medios, por otro lado, observan con mucha atención qué palabras emplean los actores en la arena política, para así poder alabar o censurar a una u otra formación.

$\mathrm{Y}$ es precisamente en este marco donde en el ámbito germano-hablante, y más especialmente en Alemania y Austria, el lexema VOLK ha sido objeto de innumerables estudios desde un punto de vista no solo lingüístico, sino también histórico, cultural y literario. Prueba de ello son, entre otros, los estudios de von Senger (2013), Bergem (2005), von Polenz (1999), Hildebrandt (1980), o alguno más centrado en las estrategias en el lenguaje político, como Kopperschmidt (1991). No han faltado tampoco aportaciones que ponen en contacto términos como "pueblo", "nación", "alemán" y "lengua", analizándolos a lo largo de las sucesivas épocas históricas de Alemania, como Retterath (2016), Jakobs (2000), Thomas (2000), Knape (2000), Gardt (2000a/b), Bär (2000), Lerchner (2000) y Koselleck (1992). Finalmente, Chilton ofrece una interesante aportación desde la perspectiva neuro-cognitiva, que trata de explicar "the hold of populist discourse over minds and behaviours, rather than simply describing the surface" (2017: 592). Desde su punto de vista, el discurso político busca activar conceptos con una carga emocional y valorativa, así como sus correspondientes emociones, antes que servirse de argumentaciones y evidencias patentes. En este sentido, la pregunta sería en qué medida lo que Chilton (2017: 592) considera "mere words", entre las cuales VOLK constituye un ejemplo palmario, pueden afectar a pensamientos, emociones y sentimientos.

Ahora bien, volviendo al lexema VOLK, hay una afirmación del escritor alemán Bertolt Brecht que ha sido citada en numerosas ocasiones en los más diversos ámbitos, de los cuales el discurso político no ha sido una excepción: "Wer in unserer Zeit statt Volk Bevölkerung und statt Boden Landbesitz sagt, unterstützt schon viele Lügen nicht"s. El

Citado por Kopperschmidt (1991: 70). La cita de Brecht procede originalmente de un artículo suyo titulado "Fünf Schwierigkeiten beim Schreiben der Wahrheit”), que apareció publicado en una revista antifascista, en 1934. 
término en sí ha sido objeto de numerosas discusiones e intentos de definición en los últimos años, tratando de matizar quién estaría incluido en él y quién no. Es conocida a este respecto la declaración de la presidenta alemana Angela Merkel, según la cual "Das Volk ist jeder, der in diesem Land lebt"4. No menos conocida fue la polémica que se desató ante el uso de la palabra völkisch por parte de Frauke Petry, exdirigente de la AfD 5 .

Algo parecido sucede con VOLK en el lenguaje político en lengua alemana, tanto en Alemania como en Austria. Su uso parece ser muy limitado, en algunos casos, y evitado, en otros. Esto hace que en la actualidad compita fuertemente con BEVÖLKERUNG, que no arrastra tanta polémica ni cuenta con las connotaciones negativas que, sobre todo por motivos históricos, se suelen atribuir a VOLK. En un principio existen indicios de que se trata de un término restringido a los denominados partidos populistas, concretamente, a la AfD en Alemania y al FPÖ en Austria, que parece constituir una marca de identidad política y social de una formación o, en términos más generales, de una determinada ideología política. Ahora bien, el uso del lexema VOLK en el lenguaje político plantea algunos interrogantes, que constituyen el objeto de estudio del presente trabajo: ¿son realmente los denominados "partidos populistas" los únicos en emplear este lexema? Si la respuesta a esta pregunta fuera negativa, ¿tiene el mismo significado y referente en todas las formaciones políticas que lo emplean? En tal caso, ¿qué desean comunicar realmente unos y otros cuando utilizan el lexema VOLK? Aparte de eso, ¿se observa algún tipo de comportamiento similar en Alemania y en Austria en cuanto a su uso?

\section{Metodología}

El análisis se centra en los programas de los diferentes partidos para las elecciones generales del año 2017, tanto en Alemania como en Austria. Para ello fueron elaborados dos corpora textuales independientes, uno para Alemania y otro para Austria, con los programas mencionados compilados por la herramienta digital Sketch Engine $^{6}$.

Se llevó a cabo un estudio de corpus en el que se analizó el número de veces que aparecía VOLK en cada programa electoral, la frecuencia de aparición en relación al número total de palabras de cada programa, y, finalmente, se compararon los resultados de todos los partidos, con objeto de ofrecer al lector una visión global de uso. También se llevó a cabo un análisis contextualizado de cada aparición de VOLK, que permitiera conocer el significado referencial con el que es usado por cada partido, en

4 Cfr. Berliner Zeitung, del 23 de febrero de 2017: http://www.berliner-zeitung.de/politik/merkel-zu-pegida-rufen-das-volk-ist-jeder--der-in-diesem-land-lebt--25820352 [13/10/2017].

5 Cfr: "Petry will Begriff 'völkisch' positiv besetzen", en Süddeutsche Zeitung, http://www.sueddeutsche.de/politik/ alternative-fuer-deutschland-petry-will-begriff-voelkisch-positiv-besetzen-1.3156403, y en Die Welt, Balzli, B./ Kamann, M., "Petry will ,völkisch“ positiv besetzen", https://www.welt.de/politik/deutschland/article158049092/ Petry-will-den-Begriff-voelkisch-positiv-besetzen.html, del 11 de septiembre de 2016 [13/10/2017]; "Petry sieht 'völkisch' als positiven Begriff', en Frankfurter Allgemeine Zeitung, del 11 de septiembre de 2016, http://www. faz.net/aktuell/politik/inland/afd-petry-sieht-voelkisch-als-positiven-begriff-14430103.html [13/10/2017]; Biermann, K., “'Völkisch' ist nicht irgendein Adjektiv”, en Zeit Online, del 11 de septiembre de 2016, http://www.zeit. de/kultur/2016-09/frauke-petry-afd-voelkisch-volk-begriff-geschichte [13/10/2017].

6 Enlace a la herramienta: https://the.sketchengine.co.uk/login [16/10/2017]. 
el caso de constatar que había más de un partido en cada país que lo empleara. Ello permitió comprobar, también de modo contrastivo, si los diferentes partidos utilizan VOLK con el mismo contenido semántico y referencial.

Hasta aquí, el análisis individualizado por países, primero en Alemania y, posteriormente, en Austria. A continuación se efectuó un análisis contrastivo, comparando los resultados en ambos estados, a fin de constatar o no un comportamiento similar en cuanto al uso de VOLK en ambos escenarios electorales, siempre centrados en los respectivos programas electorales.

El presente estudio tiene, en uno de sus aspectos, un carácter cuantitativo, puesto que trata de averiguar cuántos partidos se sirven del lexema VOLK y si este aparece solo marginalmente o si, por el contrario, muestra una presencia considerable. También se trata de ver si, en el caso de detectar su uso en más de un partido en Alemania y Austria, respectivamente, este se produce de manera equilibrada o con grandes diferencias. Por otra parte, el trabajo realizado también tiene un carácter cualitativo, ya que trata de dilucidar, siempre y cuando el empleo de VOLK sea una realidad en más de un partido, hasta qué punto se manejan los mismos matices semánticos y referenciales, o si, por el contrario, se utiliza en cada caso para referirse a realidades diferentes.

Los programas electorales fueron obtenidos directamente en las páginas web de los respectivos partidos políticos, en formato $\mathrm{PDF}$, y cargados posteriormente al banco de datos de la herramienta Sketch Engine. En cuanto al análisis de VOLK, hay que decir que solo se analizó este lexema, tanto en su realización en singular (Volk) como en plural (Völker). No fueron tenidos en cuenta términos derivados (por ej. Bevölkerung), ni compuestos (por ej. Volksschule).

En una fase posterior al estudio de corpus, se intentará aquí desarrollar el marco argumentativo subyacente, que puede estar sirviendo de cimentación a los partidos populistas a la hora de usar VOLK, de igual manera que a los demás partidos, para evitarlo o, en caso de usarlo, hacerlo con un significado referencial distinto. Aquí serán de utilidad estudios previos en torno al concepto de VOLK desde una perspectiva lexicográfica, histórica y, por supuesto, también del lenguaje político.

\section{Corpus de VOLK en los programas electorales de 2017 y su análisis}

\subsection{VOLK en programas electorales alemanes}

El corpus consta de los programas de las siguientes formaciones políticas: Die Grünen, Die Linke, Sozialdemokratische Partei Deutschlands (SPD), Freie Demokratische Partei (FDP), Christlich Demokratische Union Deutschlands (CDU) y Alternative für Deutschland (AfD).

Tras localizar todas las muestras de VOLK, en cualquier forma flexiva, el resultado puede verse en el cuadro 1.

El cuadro 1 ofrece una visión panorámica que permite comprobar qué partidos emplean el lexema VOLK en Alemania. En este sentido, hay que destacar que, de 6 partidos, más de la mitad usan VOLK, entre los que se encuentra un partido mayoritario, como la SPD. La CDU y el FDP, por el contrario, no lo emplean en absoluto. También resulta llamativo comprobar que la AfD usa este le- 
xema en 17 ocasiones, es decir, por encima de 8 veces más que los siguientes: SPD y DIE LINKE.

\section{№ global muestras}

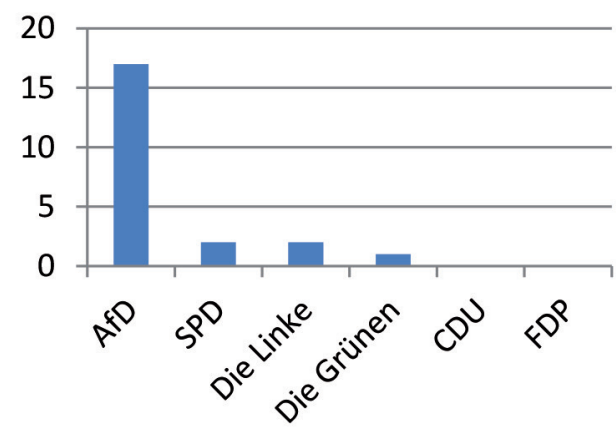

№ global muestras

Cuadro 1. Número total de muestras de VOLK en cada programa electoral.

Con todo, los resultados que presenta el cuadro 1 son relevantes, pero insuficientes, ya que sería conveniente también saber qué peso específico tiene la presencia de VOLK en cada programa electoral. Para este fin, fueron calculados los tantos por mil en relación al número total de palabras en cada programa electoral, cuyos resultados se presentan a continuación, en la tabla 1:

Tabla 1. N. ${ }^{\circ}$ total de palabras y tantos por mil (\%o) de la frecuencia de VOLK en cada programa.

\begin{tabular}{|l|c|c|}
\hline \multicolumn{1}{|c|}{ Formación política } & $\mathbf{N .}^{\mathbf{0}}$ palabras programa electoral & \%o \\
\hline AfD & 17.095 & 0,99 \\
\hline SPD & 37.003 & 0,05 \\
\hline DIE LINKE & 59.498 & 0,03 \\
\hline DIE GRÜNEN & 63.617 & 0,01 \\
\hline FDP & 35.987 & 0,0 \\
\hline CDU & 19.472 & 0,0 \\
\hline
\end{tabular}

Ahora bien, estos resultados preliminares precisan de un análisis más detallado del corpus, en el que las diversas muestras aparecen en sus contextos oracionales. Ello nos permitirá comprobar si todas las formaciones políticas analizadas están hablando exactamente de lo mismo cuando usan VOLK. Para este fin, se tuvo en cuenta la oración en la que aparece VOLK en su totalidad, atendiendo también a las colocaciones de este lexema. 
Al observar detenidamente cada una de las citas, se comprueba lo siguiente: todos los partidos, a excepción de la AfD, utilizan VOLK para referirse exclusivamente a "pueblos" o etnias en general, entendidos estos como comunidades de personas con unas características comunes, pero sin referirse en ningún caso al pueblo alemán, siendo este el rasgo más destacable. Así, mientras DIE GRÜNEN usan VOLK para referirse a los judíos y a los habitantes del estado de Israel, la SPD y DIE LINKE se refieren a los "pueblos" en general, si bien DIE LINKE también los individualiza y les pone nombre: comunidades dentro de la Unión Soviética, gitanos, judíos, etc. Véanse a modo de ilustración las citas siguientes:

1. Wir GRÜNE setzen uns weiterhin für eine Zwei-Staaten-Regelung ein, um den Fortbestand des Staates Israel als nationale Heimstätte des jüdischen Volkes und zum Wohle aller seiner Bewohner*innen sowie die Schaffung eines souveränen, lebensfähigen und demokratischen Staates Palästina auf der Grundlage der Grenzen von 1967 zu gewährleisten (DIE GRÜNEN, Programa Electoral 2017, 82).

2. Die europäische Einigung ist ein einzigartiger historischer Erfolg. Sie beruht auf der Idee des freien, friedlichen und solidarischen Zusammenlebens der Menschen und Völker Europas (SPD Programa Electoral 2017, 94-95).

3. Deutschland hat wegen der beispiellosen Verbrechen der Deutschen an den Jüdinnen und Juden, wegen der Entfesselung des Zweiten Weltkrieges und den daraus resultierenden Verbrechen an den Völkern Europas - besonders massenhaft an den Völkern der Sowjetunion, an den Sinti und Roma, an politisch Andersdenkenden, behinderten Menschen und Homosexuellen eine besondere Verantwortung und muss jeder Art von Menschenfeindlichkeit, Anti-semitismus, Antiziganismus und Rassismus entgegentreten (DIE LINKE, Programa Electoral 2017, 110).

Ahora bien, en lo que respecta a la AfD, se constatan dos actitudes en cuanto al uso de VOLK: por un lado, hay ocasiones en las que lo emplea de manera similar al resto de partidos, refiriéndose a pueblos en general o a unos muy concretos, como el francés y el holandés, es decir, sin hacer alusión al alemán, como se puede observar en: "Das ist die Lehre aus der Wiedervereinigung, aus der Ablehnung des 'EU-Verfassungsvertrags' durch das französische und das niederländische Volk und der Entscheidung der Engländer, aus der Lissabon-Union auszutreten" (AfD, Programa Electoral 2017, 9).

Pero el verdadero contraste entre la AfD y los demás partidos que utilizan el lexema VOLK se produce en la mayor parte de las restantes citas. En estas citas, la AfD hace alusión directa (cita 4) o indirecta (cita 5) al pueblo alemán:

4. Das deutsche Volk soll deshalb nach dem britischen Vorbild über den Verbleib Deutschlands in der Eurozone und gegebenenfalls der EU abstimmen! (AfD, Programa Electoral 2017, 8).

5. Wir wollen den Bundespräsidenten deshalb direkt durch das Volk wählen lassen (AfD, Programa Electoral 2017, 10).

En la cita 4 VOLK aparece junto al adjetivo deutsch (“alemán”), mientras que en la cita 5 , sin hablarse explícitamente del pueblo alemán, VOLK aparece, sin embar- 
go, en una misma oración con el pronombre personal wir ("nosotros"), cuyo contenido referencial aquí es indudablemente la AfD, así como también con Bundespräsident, de manera que, aun sin expresarlo explícitamente, se puede entender sin lugar a dudas una alusión al pueblo alemán, ya que ¿quién si no podría elegir legítimamente al Bundespräsident en Alemania? De las citas de los demás partidos, por el contrario, no es fácil, por no decir imposible, deducir una referencia directa o indirecta al pueblo alemán.

Otro aspecto que llama la atención en el corpus es la consideración de los "pueblos de Europa" y de un "pueblo europeo", sobre todo en relación con la UE. Tanto la AfD como los demás partidos no parecen tener problema alguno en hablar de "pueblos europeos", entre los que también se encuentra sin duda el alemán:

6. Die Völker Europas werden diese Entwicklung nicht klaglos hinnehmen und sich zu Recht gegen die politischen Eliten auflehnen (AfD, Programa Electoral 2017, 14).

7. Die europäische Einigung ist ein einzigartiger historischer Erfolg. Sie beruht auf der Idee des freien, friedlichen und solidarischen Zusammenlebens der Menschen und Völker Europas (SPD, Programa Electoral 2017, 94-95).

Pero hay que decir que en este contexto de "pueblos europeos" la AfD es muy explícita a la hora de afirmar que esto no se puede llegar a confundir nunca con un "pueblo europeo único": "Es gibt weder ein europäisches Staatsvolk, das für ein solches Vorhaben konstitutiv wäre, noch ist erkennbar, dass sich ein solches auf absehbare Zeit herausbildet" (AfD, Programa Electoral 2017, 7).

Con ello, la AfD se está oponiendo a la idea ocasional de un "pueblo europeo" homogeneizado, en el sentido de que las identidades individuales, entre ellas la alemana, se diluyan a favor de algo genérico y uniformizante. Una cosa es, por ejemplo, la unidad monetaria, y otra muy distinta es el hecho de ignorar sin más los rasgos propios e insustituibles de una identidad nacional, cultural, lingüística, etc.

Así pues, queda patente la diferencia en el uso de VOLK por parte de los principales partidos políticos alemanes: mientras hay partidos que no lo usan en absoluto (CDU, FDP), hay otros que lo usan, pero sin referirse nunca a Alemania ni a sus habitantes, sino más bien a poblaciones, comunidades o grupos de población minoritarios o perseguidos en algún momento de la historia más o menos reciente ${ }^{7}$. En cambio, únicamente la AfD lo emplea mayoritariamente para referirse de forma explícita e implícita a Alemania y a los alemanes.

\subsection{VOLK en programas electorales austríacos}

Antes de proceder al análisis de los programas electorales austríacos resulta oportuno llamar la atención sobre las diferencias de Austria respecto de Alemania. Estas pueden ser importantes a la hora de contextualizar el uso de VOLK, ya que, aun tratándose de dos estados que tienen el alemán como lengua nativa oficial, la historia de estos dos países ha tenido, sin embargo, sus propios derroteros, especialmente durante los dos últimos siglos. Un análisis histórico detallado aquí se saldría, cierta-

Se debe tener en cuenta que en España, el término "pueblo" tiene un carácter polisémico y que, en ocasiones, se prefiere utilizar otros como "ciudadanía" o "etnia". 
mente, de los objetivos del presente trabajo, sin embargo, parece proporcionado llamar la atención brevemente sobre el papel de ambos países, especialmente durante el periodo del Nacionalsocialismo, a modo de ilustración muy concisa.

Austria se vio involucrada en cierta medida en el Nacionalsocialismo, al ser anexionada al III Reich en 1938, sin embargo, nada más terminar la Segunda Guerra Mundial la "anexión" (Anschluss) fue declarada nula mediante la Proclamación de Independencia de Austria del 27 de abril de 1945, según la cual la anexión se había producido bajo amenazas y presiones, y había sido impuesta abusivamente en contra de los intereses de Austria, en lugar de servir a los intereses de ambos estados ${ }^{8}$.

En este sentido, tras el fin de la Segunda Guerra Mundial los austríacos se sentían más liberados que vencidos, al contrario de lo que sucedía en Alemania, que quedaba como la gran derrotada y con la responsabilidad de reparar los daños de guerra y los crímenes contra la humanidad, especialmente por el denominado Holocausto. Si se lee el texto de la Proclamación, abundan expresiones como "ocupación, amenaza, degradación, desvalijamiento", etc., así como de una anexión conseguida mediante una "amenaza militar desde fuera y el terror altamente traicionero de una minoría nazi y fascista",

Así pues, Alemania tuvo que someterse a un proceso de "desnacificación" tras la guerra, mientras que en Austria dicho proceso no tuvo lugar, algo que se manifiesta, entre otros factores, en el hecho de que, estando aún tan reciente la guerra, los firmantes de la Proclamación no tienen reparo en emplear con frecuencia considerable el lexema VOLK, para referirse al "pueblo austríaco", mientras que en Alemania se tiende a evitar su uso, dada su presencia en el discurso nacionalsocialista. Para ser más exactos, VOLK aparece un total de 23 veces en un texto de 2598 palabras, con una frecuencia de 8,88 \%o. De estas 23 muestras, al menos 18 se refieren directa o indirectamente a Austria.

En consecuencia, ambos estados muestran una sensibilidad diferente ante el recuerdo de la Segunda Guerra Mundial y el Nacionalsocialismo, y, por lo tanto, no debe extrañar que ello se manifieste también de algún modo en el discurso político, como puede ser el uso de determinadas expresiones debido a la asociación más o menos directa que se pueda establecer con episodios negativos de un pasado aún no muy lejano. Y el uso de una expresión como VOLK, así como su significado referencial, es un ejemplo de ello.

Ahora bien, volviendo al análisis de VOLK en el ámbito austríaco durante las últimas elecciones, el corpus consta de los programas electorales de las siguientes formaciones políticas: Österreichische Volkspartei (ÖVP), Sozialdemokratische Partei Österreichs (SPÖ), Grünen, Freiheitliche Partei Österreichs (FPÖ), Das Neue Österreich und Liberales Forum (NEOS).

La búsqueda de muestras de VOLK en los diferentes programas electorales arroja los siguientes resultados, que se presentan en el cuadro 2.

Como se puede observar en el cuadro 2, solo hay dos partidos que usan el lexema VOLK en sus programas. En cuanto a los partidos que lo emplean, a saber, el FPÖ y la ÖVP, la diferencia es nula, desde un punto de vista estrictamente cuantitativo.

\footnotetext{
$8 \quad$ Cfr. Texto de la Proclamación en: Staatsgesetzblatt für die Republik Österreich, https://www.ris.bka.gv.at/Dokumente/BgblPdf/1945_1_0/1945_1_0.pdf [11/01/2018].

$9 \quad$ Ibid., p. 1.
} 


\section{№ global de muestras}

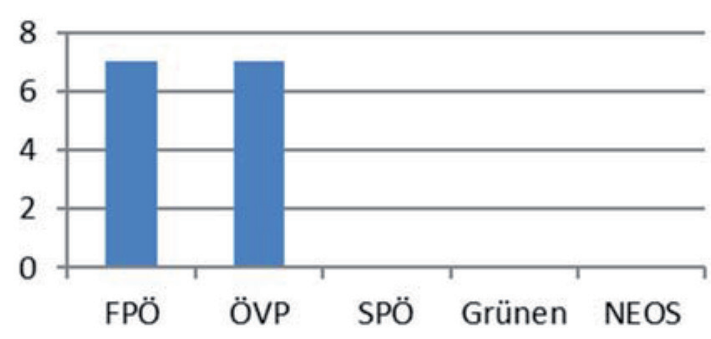

№ global de muestras

Cuadro 2. Número total de muestras de VOLK en cada programa electoral.

En cuanto a la presencia del lexema VOLK en cada programa, en relación al número total de palabras, el cálculo arroja los resultados reflejados en la tabla 2:

Tabla 2. N. ${ }^{o}$ total de palabras y tantos por mil (\%o) de la frecuencia de VOLK en cada programa.

\begin{tabular}{|l|c|c|}
\hline \multicolumn{1}{|c|}{ Formación política } & $\mathbf{N}^{\mathbf{0}}$ palabras programa electoral $^{-}$ & $\mathbf{\% o}$ \\
\hline FPÖ & 7.743 & 0,9 \\
\hline ÖVP & 54.440 & 0,12 \\
\hline SPÖ & 51.542 & 0,0 \\
\hline Grünen & 18.053 & 0,0 \\
\hline NEOS & 1.778 & 0,0 \\
\hline
\end{tabular}

A la vista de los resultados de la tabla 2 se puede comprobar que, si bien el FPÖ y la ÖVP coinciden en el número de muestras de VOLK en sus respectivos programas, la relevancia de dichas muestras no es, en cambio, la misma. En términos absolutos, la presencia de VOLK es con diferencia cuantitativamente mayor en el programa del FPÖ que en el de la ÖVP, debido ante todo a que el programa de la ÖVP es casi 7 veces más extenso, en número de palabras, que el del FPÖ.

Con todo, una vez contemplados los resultados globales, se hace necesario llevar a cabo un análisis más detallado en cuanto al uso de VOLK. Al igual que en el caso de los partidos alemanes, se trata de comprobar si el FPÖ y la ÖVP están hablando o no de lo mismo al servirse de este lexema.

En primer lugar, hay que decir que ambos partidos se sirven de VOLK para hacer referencia tanto a Austria, al pueblo austríaco, como a los pueblos de Europa. Ahora bien, un análisis más profundo saca a la luz algunas diferencias. Así, por ejemplo, si bien ambas formaciones hacen alusiones indirectas al pueblo austríaco: "Dass über das Volk hinweg entschieden wird und Wahlversprechen nicht eingelöst werden" (FPÖ, Programa Electoral 2017, 10), "Politik für das Volk und durch das Volk" 
(ÖVP, Programa Electoral 2017, III, 38), la ÖVP es, sin embargo, la formación que hace afirmaciones donde se habla más explícitamente de lo austríaco, como se puede constatar en las citas siguientes: "Mit der österreichischen Verfassung bekennen wir uns zu einer demokratischen Republik, deren Recht vom Volk ausgeht" (ÖVP, Programa Electoral 2017, III, 14); "Eine Volksbefragung, in der das Volk unverbindlich $\mathrm{zu}$ einem Thema befragt wird, gab es bisher überhaupt nur ein einziges Mal, und zwar 2013 zur allgemeinen Wehrpflicht ${ }^{10 "}$ (ÖVP, Programa Electoral 2017, III, 37).

Ciertamente, en estas dos últimas citas de la ÖVP no se menciona a Austria ni el adjetivo "austríaco" junto a VOLK, pero sí aparecen en la misma oración elementos que hacen referencia inequívoca a Austria. En la primera encontramos la mención de la Constitución de Austria, y en la siguiente la referencia a la consulta popular realizada en 2013, acerca de si se debía eliminar el servicio militar obligatorio en el país.

El FPÖ, sin embargo, al hablar de VOLK focaliza más en su programa los rasgos autóctonos y étnicos, lo cual sirve sin duda para el pueblo austríaco:

8. In einer Zeit der Identitätsvernichtung und der Entfremdung der Völker von ihren Wurzeln im Interesse globaler Großkonzerne und weltweit tätiger Finanzjongleure gilt dem ideellen Engagement für die Bewahrung der eigenen Kultur und Sprache besondere Achtung (FPÖ Programa Electoral 2017, 7)

Si se contrasta esta declaración del FPÖ con las de la ÖVP en torno a VOLK, queda claro que en las de la ÖVP, por mucho que hagan alusión al pueblo austríaco, su contenido referencial no es tan restringido y específico. Dicho con otras palabras, de la cita del FPÖ sobre VOLK se puede deducir que lo autóctono (lengua, cultura, territorio, historia, etc.) es lo que termina conformando el contenido semántico y referencial de VOLK con todas sus consecuencias: al "pueblo austríaco" pertenecería, entonces, aquel que posee los rasgos autóctonos. En el discurso de la ÖVP, por el contrario, lo autóctono no está tan presente, de modo que, aunque el adjetivo "austríaco" aparezca cerca del lexema VOLK, dicho adjetivo puede incluir entre sus miembros a individuos que no comparten un mismo origen de nacimiento, cultura, lengua e, incluso, religión.

Con todo, también resulta ilustrativo el análisis cuando se analiza VOLK en combinación con europäisch ("europeo") o con la Unión Europea, algo que hacen ambos partidos:

9. Die FPÖ steht für ein Europa als Verbund von selbstbestimmten Völkern und Vaterländern, die in der Tradition einer abendländischen geprägten Leitkultur stehen (FPÖ, Programa Electoral 2017, 50).

10. Wir müssen auch die Strukturen verschlanken, die Kommission verkleinern und sollten die Präsidentin oder den Präsidenten der Europäischen Kommission direkt vom Volk wählen lassen" (ÖVP, Programa Electoral 2017, III, 42).

Además, llama especialmente la atención el hecho de que la ÖVP emplea VOLK únicamente en singular, mientras que el FPÖ lo hace también en plural. Ahora bien, centrando el análisis en su uso en conexión con Europa o la Unión Europea, la ÖVP

10 Cfr. Republik Österreich Bundesministerium für Inneres: Volksbefragung 2013. URL: http://vb2013.bmi.gv.at [19/10/2017]. 
usa VOLK en singular exclusivamente, mientras que el FPÖ lo hace en plural. Este es un matiz que no debería ser pasado por alto, y cuya explicación podría ser la siguiente: por un lado, la ÖVP es un partido a favor de Europa y de la Unión, y un modo de dar expresión a esta idea es hablar de una comunidad unida, dejando en un segundo plano — siempre desde una perspectiva lingüística - las particularidades autóctonas de cada pueblo que integra dicha comunidad. Con ello no se tiene que estar buscando necesariamente diluirlas de un modo consciente, sino que más bien se resalta y se pone por delante el deseo de una unión o comunidad que trasciende lo local o, si se prefiere, lo autóctono, sin ver en ello algo incompatible.

El FPÖ, por el contrario, en el mismo contexto no habla de "un pueblo europeo" sino de "pueblos europeos". En el discurso de este partido, de carácter claramente etnonacionalista ${ }^{11}$, se busca que cada pueblo esté y permanezca en su lugar o territorio de origen. En consecuencia, el FPÖ parece proyectar el miedo de que la inercia política lleve a que la identidad popular, cultural y lingüística propia e individual de cada uno de sus integrantes quede finalmente muy difuminada, a favor de una homogeneización impuesta desde fuera por un exceso de centralismo, algo sobre lo que también llama la atención la AfD de forma muy clara en Alemania (Wildt 2017: 97). Y un modo de llamar la atención sobre ello y evitarlo, es, precisamente, expresándose lingüísticamente en términos de "[...] una Europa como alianza de pueblos y patrias autónomos inmersos en una tradición cultural occidental"12.

\section{Discusión en torno del uso de VOLK en Alemania y Austria}

Por último, y desde un punto de vista comparativo entre los resultados obtenidos de programas electorales alemanes y austríacos, cabe destacar una serie de concomitancias y diferencias. En primer lugar, tanto la AfD y el FPÖ ponen el énfasis en una identidad autóctona de lo alemán y de lo austríaco, respectivamente. Ambas formaciones rechazan de un modo más o menos explícito todo aquello que en el discurso pudiera conducir, aunque solo sea a un nivel puramente conceptual, a una idea de "pueblo" europeo como unidad supranacional, en detrimento de los llamados "pueblos autóctonos" caracterizados por una lengua, cultura, costumbres, etc. singulares y diferenciadoras. En otras palabras, estos partidos pretenden evitar que lo autóctono quede diluido ante lo europeo y que, en consecuencia, el contenido semántico de VOLK no pueda referirse a un "pueblo europeo" uniforme.

En segundo lugar, mientras la AfD antepone el adjetivo deutsch a Volk, o bien el posesivo de primera persona de plural unser, el FPÖ sigue otra estrategia: las apariciones de Volk están siempre enmarcadas en secciones cuyo encabezado incluye en el $100 \%$ de las veces ${ }^{13}$ el posesivo unser, por lo que la identificación de Volk con Austria es difícil de pasar por alto. Y, además de esto, si bien no habla del "pueblo austríaco", sí antepone un número considerable de veces el adjetivo österreichisch ${ }^{14}$ a sustantivos que hacen alusión a diversos grupos de personas que constituyen dicho

11 Como introducción al concepto de "etnonacionalismo", puede consultarse, entre otros: Connor, W., Ethnonationalism: the quest for understanding, 1994 y Conversi, D., Ethnonationalism in the contemporary world: Walker Connor and the study of nationalism, 2002.

12 Cfr. Programa electoral, p. 50.

13 El número total de secciones en el programa electoral es 25.

14 Es decir, 32 veces, un $0,31 \%$ del total del corpus. 
pueblo austríaco, ya sea el criterio la edad (österreichische Kinder), como la profesión/gremio (österreichische Maturanten, österreichische Jäger), o situación social (österreichische Arbeitnehmer, österreichische Steuerzahler), etc.

En cuanto al uso de Volk en sí, el corpus analizado muestra que tanto la AfD como el FPÖ no son las únicas formaciones que lo emplean. Ahora bien, desde una perspectiva estrictamente cuantitativa hay que constatar dos diferencias: por un lado, en Alemania cuatro de seis partidos conocidos que concurren a las elecciones usan Volk $(>50 \%)$, mientras que en Austria solo lo hacen dos de cinco $(<50 \%)$ partidos; por otro lado, en Alemania, la AfD encabeza la lista en número de veces que usa Volk, con una frecuencia ocho veces por encima del siguiente partido, mientras que en Austria el FPÖ se halla en una situación de empate numérico con la ÖVP.

En cuanto a esto último, si tenemos en cuenta la perspectiva neuro-cognitiva de Chilton (2017), lo de menos es el aspecto cuantitativo, sino más bien cómo cada uno de estos dos partidos "controla" el significado de la palabra, en este caso Volk. Cada una de estas formaciones tiene su modo de conceptualizar y evaluar la realidad a la que quiere hacer referencia mediante Volk. Una vez más, es llamativo que al colocar Volk cerca de Europa, el FPÖ hable de europäische Völker, mientras que la ÖVP habla de europäisches Volk. Esto confirma su tesis, según la cual "There are no guarantee that the meanings ${ }^{15}$ of identical lexemes are the same" (Chilton 2017: 587). Cada partido "controla" o "manipula" el significado de un mismo lexema, dentro de unos límites más o menos amplios, buscando una reacción concreta en los receptores de sus respectivos discursos.

\section{Posible marco para el uso u omisión de VOLK por parte de los partidos políticos}

Tras analizar el corpus, y antes de pasar a las conclusiones de este estudio, puede ser oportuno tratar de averiguar si el uso u omisión de VOLK por parte de las diferentes formaciones políticas, tanto alemanas como austríacas, puede estar motivada o guarda algún tipo de relación con factores socio-históricos del propio lexema. Con ello no se trata, en absoluto, de realizar aquí un estudio exhaustivo sobre el lexema VOLK, aparte de que a día de hoy ya existen muchos, sino más bien de lo siguiente: basándonos en estudios ya existentes sobre VOLK, el objetivo ahora consistiría en ver en qué aspectos socio-históricos de VOLK han podido servir de base a los diferentes partidos políticos para servirse de él o evitarlo en su discurso, y más concretamente, en sus programas electorales. Y aquí entran en juego tanto estudios teóricos como diccionarios, ya que estos últimos son utilizados a veces como puntos de partida (Hermanns 2003; Hoffmann 1991).

En primer lugar, no hay que dejar de insistir en que un término como Volk ha desatado acaloradas discusiones y numerosos estudios, también en la actualidad política, con motivo de los llamados "populismos" (Wildt 2017; Hermanns 2003; Lötzsch 1999; Hoffmann 1991). Y, dentro de esta misma discusión, un aspecto que se ha estado mirando con especial cuidado es el de la denominada "pertenencia", es decir, la pregunta quién pertenece a un "pueblo" determinado y quién queda excluido (Wildt 2017; Hermanns 2003; Müller 1997; Hoffmann 1991).

15 La cursiva es del original. 
También es interesante la observación que se hace en este contexto acerca de los criterios de pertenencia a un pueblo. Así, Wildt (2017:112ss.), analizando el caso concreto de la AfD en Alemania, destaca que la definición de Volk se lleva a cabo desde un perspectiva negativa cuando para ello se hacen afirmaciones del tipo "Der Islam gehört nicht zu Deutschland"16, porque de este modo se construye una definición a base de nombrar a los que no pertenecen a dicho Volk.

Ahora bien, como decía más arriba, se trata ahora de exponer en la medida de posible de dónde pueden proceder los marcos argumentativos de partidos como la AfD y el FPÖ, para usar el lexema VOLK.

Uno de ellos lo podemos encontrar en uno de los antiguos significados de Volk, recogido en el diccionario de Adelung (1793-1801), según el cual Volk significa las clases sociales, las cuales se alimentan de su propio trabajo manual, el pueblo común, el gentío o la masa ${ }^{17}$. Según el propio Adelung, hubo intentos de "ennoblecer" la palabra y su uso ${ }^{18}$, algo que nos recuerda en cierto modo la pretensión de F. Petry con völkisch, término que igualmente pretendía rehabilitar en el lenguaje político ${ }^{19}$.

Asimismo, el término Volk ha estado ligado también a matices degradantes y de menosprecio social, como apuntan los hermanos Grimm en su Deutsches Wörter$b_{u c h}{ }^{20}$. Este es un aspecto que ha estado presente más tarde en otros estudios (Wildt 2017; Priester 2012; Hermanns 2003; Lötzsch 1999), en los que el "pueblo" era considerado Pöbel, es decir, la "chusma/gentuza", de la cual la clase gobernante estaba separada y se desentendía. A esto último se refiere también Adelung (17931801IV: 1226), al añadir el matiz de que el "pueblo" era todo aquello que no abarcaba a los superiores o gobernantes, que son, paradójicamente, los que toman las decisiones que le afectan.

Esto último es lo que puede haber debajo de la postura de la AfD, cuando afirma que el "pueblo alemán" es suficientemente maduro y que se le debe devolver la soberanía. Tampoco se pueden tomar decisiones sin contar con él ni engañarle, como también da a entender el FPÖ.

Ahora bien, leyendo bibliografía previa en torno al concepto de VOLK, se puede llegar a entender matices en torno a quién pertenece realmente a dicho VOLK y quién no. Esto es algo que también ha ocupado su espacio en la discusión en torno a este concepto (Wildt 2017; Hermanns 2003; Wiktorowicz 2002; Müller 1997; Berbig 1976).

Con todo, la clave está en por qué Volk fue utilizado con mucha frecuencia en el periodo nacionalsocialista en Alemania. Como destaca Wiktorowicz (2002: 347), durante dicho periodo de la historia alemana se usa Volk casi exclusivamente, mientras que otros términos como Nation se evitan. Y es que Volk tenía entonces una carga semántica que apuntaba muy unívocamente a una comunidad de

16 La afirmación es atribuida a la AfD. Cfr. Die Zeit online: http://www.zeit.de/news/2016-05/01/parteien-afd-praesentiert-sich-als-deutschnationale-partei-01083004 [24/10/2017]; Handelsblatt: http:/www.handelsblatt.com/politik/ deutschland/beatrix-von-storch-der-islam-gehoert-nicht-zu-deutschland/13848914-3.html [24/10/2017]; Frankfurter Allgemeine Zeitung: http:/www.faz.net/aktuell /feuilleton/warum-satz-islam-gehoert-nicht-zu-deutschland-sinnlosist-14220186.html?printPagedArticle =true\#pageIndex_0 [24/10/2017]; Süddeutsche Zeitung: http://www.sueddeutsche.de/politik/afd-parteitag-afd-beschliesst-anti-islam-kurs-mit-grosser-mehrheit-1.2975205 [24/10/2017].

17 Cfr. Adelung (1793-1801), vol. IV, col. 1226; también Wiktorowicz (2002); Hermann Bausinger (1991: 170).

18 Ibid.

19 De los intentos de ennoblecer el significado y uso de VOLK habla también Wiktorowicz, op. cit., pp. 344s.

20 URL: http://woerterbuchnetz.de/cgi-bin/WBNetz/wbgui_py?sigle=DWB\&mode=Vernetzung\&lemid=G V09940\#XGV09940 [24/10/2017]. 
personas, donde matices como lo étnico, lo lingüístico, cultural y político eran las señas de identidad. Pero, sobre todo, lo que más se destacaba de Volk era el matiz étnico o, si se prefiere, el matiz de raza. Con ello, la pregunta quién forma parte del "pueblo alemán" y quién no va quedando contestada: aquel que tiene una serie de rasgos de sangre y físicos. Ahora bien, tampoco hay que olvidar que en el tiempo nacionalsocialista, la raza (Rasse), además de definirse por la sangre, también se definía políticamente, lo cual explica, por ejemplo, la exclusión de los comunistas.

Así pues, y dado que el Nacionalsocialismo, el Holocausto y la Segunda Guerra Mundial son acontecimientos aún muy frescos en la memoria de Alemania, Austria y del resto de Europa, que, además han dejado con razón recuerdos muy negativos, es de entender que en el marco político actual el uso de Volk despierte ciertas reticencias (Bausinger 1991: 169) ${ }^{21}$, salvo si se hace acompañándolo de adjetivos como französisch, spanisch, amerikanisch, etc. (Wiktorowicz 2002: 347). A esto hay que sumar, al menos a la hora de expresarse en público, la enorme sensibilización actual en contra de la xenofobia como actitud discriminatoria, incluso ya antes de que la polémica en torno al populismo creciera profusamente en los últimos años.

Aun así, hay que decir en honor a la verdad que Volk no parece, en sí mismo, racista ni discriminatorio, si se tienen en cuenta las definiciones proporcionadas por diccionarios de la lengua alemana ${ }^{22}$. Ahora bien, como todo, unos y otros pueden servirse de él para unos fines concretos, legítimos o no, lo cual les puede llevar a resaltar más unos matices sobre otros, o bien incluso manipularlos. Y esto es precisamente lo que hace que el significado de algunas palabras, a lo largo de la historia, se vea degradado o ennoblecido. En este sentido, cuando se mira el significado básico y común de Volk, tanto diccionarios como otros estudios lo definen como una comunidad cuyos elementos constitutivos son un mismo territorio, origen, procedencia, lengua, cultura, religión, costumbres e historia (DWDS ${ }^{23}$; Hermanns 2003: 27; Wiktorowicz 2002: 347; Hoffmann 1991: 195; Berbig 1976: 1).

Todo ello parece definir y singularizar a una comunidad determinada. Ahora bien, el problema puede plantearse cuando esta definición se emplea para singularizarse de manera excluyente respecto de otros (Wiktorowicz 2002: 347; Lötzsch 1999: 16), actitud que puede quedarse en algo neutro, o bien llegar a una actitud discriminatoria.

Así pues, en el caso de la AfD y de la FPÖ el empleo de Volk puede verse motivado, entre otras razones, en el marco propiciado por las recientes olas migratorias hacia Europa, especialmente hacia Alemania. Ello significa la llegada de muchas personas de una procedencia, lengua, cultura y costumbres diferentes. A ello se añade un elemento no menos importante, que es la religión, ya que muchos de estos inmigrantes son de confesión islámica.

21 Hoffmann (1991: 195), va más lejos, llamando la atención sobre la distinción entre lo que, a nivel puramente teórico, no ofrece problema alguno y un pasado cargado de antecedentes negativos, que pueden hacer inoportuno el uso de un término determinado. Luego, el problema no está en el término en sí, sino en la degradación que ha podido sufrir por diversas razones.

22 Cfr. DUDEN Universalwörterbuch (versión digital de 2011), o Wahrig Deutsches Wörterbuch (versión digital de 2011).

23 Cfr. Digitales Wörterbuch der Deutschen Sprache (DWDS). URL: https://www.dwds.de/wb/Volk [26/10/2017]. 
Todos estos elementos constitutivos han hecho que estas formaciones políticas expresen miedo ante el posible riesgo de que la cultura y la ascendencia europeas - y, más concretamente, la alemana y la austríaca, según el caso - se diluyan e incluso desaparezcan. Y parece tener, hasta cierto punto, su lógica desde una perspectiva puramente formal: si un "pueblo" se caracteriza por un territorio, una cultura, una lengua, unas costumbres, una ascendencia y una religión comunes, la llegada de millones de personas de otros territorios, culturas, costumbres, lenguas y religión resulta incompatible con dicho concepto de "pueblo", lo cual lleva a estos partidos a realizar afirmaciones como: "Wir wollen die Würde des Menschen, die Familie mit Kindern, unsere abendländische christliche Kultur, unsere Sprache und Tradition in einem friedlichen, demokratischen und souveränen Nationalstaat des deutschen Volkes dauerhaft erhalten"24, o bien "Wir sind ein Volk und keine beliebige Bevölkerung" 25 .

Esta última cita puede aportar otro elemento relevante: ¿por qué estos partidos prefieren hablar de Volk, mientras otros prefieren usar mayoritariamente Bevölkerung? Quizá un repaso de las definiciones disponibles pueda aportarnos alguna clave:

El DWDS define este concepto como "Gesamtheit der Bewohner eines bestimmten geographischen oder politischen Gebietes"26; el diccionario alemán Wahrig "Gesamtheit der Einwohner (eines Gebietes)"27; y, finalmente, el diccionario DUDEN "Gesamtheit der Bewohner und Bewohnerinnen eines bestimmten Gebiets; Einwohnerschaft"28.

A la vista de estas definiciones salta a la vista que VOLK es un concepto más restrictivo que BEVÖLKERUNG. Mientras el primero une un territorio a un tipo de personas, caracterizado por unos elementos constitutivos muy restrictivos que, a su vez, los singularizan de otros, el segundo simplemente se refiere a un grupo de personas que coinciden casi exclusivamente en la circunstancia de habitar un mismo territorio, siendo este prácticamente el único rasgo en el que se distinguen de otros grupos. Esta era, de hecho, la ya conocida definición de Volk por parte de Angela Merkel: "Das Volk ist jeder, der in diesem Land lebt"29. Por tanto, en Bevölkerung quedarían más en segundo plano elementos constitutivos como la lengua, religión, cultura, costumbres, procedencia, etc.

En consecuencia, las tendencias de unos y otros partidos quedan así bien definidas: para unos (AfD y FPÖ) hay un Wir frente a die anderen, mientras que para el resto de partidos esta diferenciación no se da, o al menos no se manifiesta. Así se explica la importancia de Volk en el discurso de la AfD y del FPÖ. No se trata simplemente de usar un término diferente de la mayoría de partidos, con la mera intención de provocar, sino más bien de usar un término que significa algo muy concreto con la intención de focalizar eso que tiene de concreto (Chilton 2017), si se me permite la repetición. En este sentido, el término Bevölkerung no resulta suficiente para partidos como la AfD y el FPÖ para dar expresión a sus propias ideas, con todas sus

\footnotetext{
Cfr. AfD, Grundsatzprogramm, 2016, p. 6.

Cfr. Twitter Frauke Petry, 03.10.2015. URL: https://twitter.com/fraukepetry [26/10/2017].

URL: https://www.dwds.de/wb/Bev\%C3\%B6lkerung [26/10/201].

Cfr. Brockkaus Wahrig Deutsches Wörterbuch. Versión digital, 2012.

Cfr. DUDEN, Universalwörterbuch. Versión digital, 2011.

Cfr. Berliner Zeitung, 26.02.2017. URL: https://www.berliner-zeitung.de/politik/merkel-zu-pegida-rufen--dasvolk-ist-jeder--der-in-diesem-land-lebt--25820352 [26/10/2017].
} 
denotaciones y connotaciones. Hermanns (2003: 26) resume todo esto del siguiente modo:

Je nachdem, wie wir uns die Welt denken, wie wir uns die Welt „vorstellen“ - je nachdem verhalten wir uns unterschiedlich. Und auch je nachdem, wie wir uns die Welt - und besonders unsere eigene Lage darin - wünschen, ist ausschlaggebend dafür, welche Art von Welt wir für uns wollen. Beides aber, ebenso das Denken wie das Wollen, drückt sich aus in Wörtern. Ohne solche Wörter, die ein (gemeinsames) Denken und ein (gemeinsames) Wollen „auf den Begriff bringen“, lässt sich (gemeinsames) historisches Handeln nicht erklären.

A este respecto, y a modo de contraste, pueden resultar ilustrativas las siguientes citas del programa electoral de la CDU:

11. Wir erwarten von allen Menschen in Deutschland, ganz gleich ob mit oder ohne Migrationshintergrund, die Achtung des Grundgesetzes und der Gesetze (p. 70).

12. $\mathrm{Zu}$ unserem Land gehören alte und neue Deutsche, Menschen mit und ohne deutschen Pass, mit und ohne Migrationshintergrund. Die große Mehrheit ebenso wie ethnische und gesellschaftliche Minderheiten (p. 70).

Llama la atención que la CDU hable en la cita 11 de todas las personas que se encuentran en Alemania, así como "A nuestro país" en 12, en lugar del "pueblo alemán" o de "nuestro pueblo". En este sentido, para este partido forma parte de Alemania aquel que se encuentra en Alemania, sin que tengan tanta importancia otros aspectos como el haber nacido también allí, hablar ya la lengua, compartir una misma tradición, cultura e historia, así como religión, que tan importantes son, en cambio, para la AfD.

Ahora bien, encontrarse físicamente en Alemania tampoco es suficiente para la CDU. Es necesario también "Wer unsere demokratische Grundordnung bekämpft, das Existenzrecht Israels ablehnt, den inneren Frieden gefährdet oder gegen Recht und Gesetz verstößt, muss mit der ganzen Härte unseres Rechtsstaates rechnen" (p. 70), así como también "Es ist in beiderseitigem Interesse, dass Integration stattfindet und gelingt" (p. 70). En este sentido, se puede observar que la CDU exige unas condiciones $^{30}$ para formar parte de Alemania (no del "pueblo alemán”), pero lo importante, a diferencia de la AfD, radica en que, una vez cumplidas dichas condiciones, ya no hay dos mundos paralelos que, no solo no se tocan, sino que tampoco puede haber trasvases entre ellos: "nosotros" vs "los otros"

En resumidas cuentas, se constatan en los diferentes discursos un conflicto de definiciones, por llamarlo de algún modo: de un lado, una definición de corte liberalconstitucional basada en el hecho de compartir ciertos valores democráticos, $\mathrm{y}$, de otro lado, una definición que implica valores culturales y, muy probablemente, aspectos de tipo étnico.

\footnotetext{
30 Dichas condiciones comprenden, entre otras, el aprendizaje de la lengua alemana y el esfuerzo por adquirir una capacitación profesional para poder subsistir autónomamente. Cfr. programa electoral, p. 74.

31 En los mismos términos se manifiesta también la FDP. Cfr. programa electoral, pp. 69s.
} 


\section{Conclusión}

En primer lugar, se constata que la AfD y el FPÖ no son los únicos partidos que, en sus respectivos estados, se sirven de Volk en sus programas electorales para 2017. No obstante, un análisis cuantitativo ha permitido constatar que, en el caso de Alemania, la diferencia de uso de este término por parte de la AfD es de ocho veces por encima de los demás partidos alemanes. En el caso de Austria, sin embargo, la diferencia entre los dos únicos partidos que emplean Volk no es, cuantitativamente, apreciable en un nivel meramente numérico. La verdadera diferencia se aprecia más bien cuando se tiene en cuenta su aparición en relación con el número total de palabras de cada programa $(0,9 \%$ FPÖ - 0,12 \% ÖVP). Y, si se compara la situación entre ambos estados, se puede comprobar que, mientras en Alemania el término Volk es usado por dos tercios de los partidos analizados, en Austria, por el contrario, su uso se limita a dos partidos de seis, es decir, a la tercera parte.

Ahora bien, desde un punto de vista cualitativo, el presente análisis ha mostrado que no siempre se está comunicando la misma realidad al emplear Volk. El contenido semántico-referencial no es el mismo cuando, en Alemania, lo utiliza la AfD que cuando lo usan los demás partidos. Y lo mismo cabe decir en Austria, entre el FPÖ y la ÖVP. Estos resultados pueden servir como indicio de que el uso u omisión de Volk en los programas electorales analizados no es algo casual, lo cual puede tener sus consecuencias a la hora de delimitar quién pertenece a un Volk determinado, y quién no.

En principio, nadie excluye abiertamente a nadie, pero si Volk se usa a conciencia, sabiendo que hace referencia a un grupo de personas con unas características muy concretas como la lengua, la religión, las costumbres, la historia y vinculadas al mismo tiempo a un mismo territorio, puede asumirse que, al menos de forma indirecta, no se considerará "propio" a quien no reúna estas características. Y lo dicho aquí puede servir también, a la inversa, aplicado a aquellas otras formaciones que, evitando el uso de Volk, son más partidarias de usar Bevölkerung. También ellas se pueden estar significando, pues con ello pueden dar a entender que para ellas el rasgo decisivo es habitar un mismo territorio.

Así pues, para partidos como la AfD y el FPÖ el uso de Volk parece ser algo decisivo, pues, a pesar de ser conscientes de la acalorada polémica en torno a su uso, para ellos parece estar en juego la identidad de ambos países, para la que el territorio simplemente no es suficiente. Con este discurso se puede estar evitando que lo autóctono, es decir, los atributos propios como la lengua, tradiciones, cultura, religión, etc. quede diluido ante la llegada más o menos masiva de personas que, de entrada, no poseen estos mismos atributos, algunos de los cuales tampoco llegarán a poseer nunca, como es el caso del nacimiento.

En cuanto al uso del lexema VOLK en sí, queda también patente que otros partidos distintos de la AfD y el FPÖ lo utilizan para referirse a grupos étnicos (judíos, gitanos) o sociales (personas discapacitadas, o de condición homosexual), pero no para Alemania ni Austria, respectivamente. Más bien, se evita utilizando otros términos como Deutschland o die Deutschen, Österreich o die Österreicherinnen und Österreicher, que son menos restrictivos, pues permiten que quien, por ejemplo, no ha nacido dentro del territorio, pueda, sin embargo, formar parte de este conjunto a partir de un momento dado e incluso obtener la nacionalidad, observando una serie de condiciones y requisitos. 


\section{Referencias bibliográficas}

Adelung, J. Ch., Grammatisches Kritisches Wörterbuch der Hochdeutschen Mundart. 17931801. URL: http://woerterbuchnetz.de/cgi-bin/WBNetz/wbgui_py?sigle=Adelung\&mode=Vernetzung\&lemid=DV01387\#XDV01387 [26/10/2017].

Alternative für Deutschland (AfD), Grundsatzprogramm 2016. URL: https://www.afd.de/ wp-content/uploads/sites/111/2017/01/2016-06-27_afd-grundsatzprogramm_web-version.pdf [26/10/2017].

Alternative für Deutschland (AfD), Programm für Deutschland. Wahlprogramm der Alternative für Deutschland für die Wahl zum Deutschen Bundestag am 24. September 2017. URL: https://www.afd.de/wp-content/uploads/sites/111/2017/06/2017-06-01_ AfD-Bundestagswahlprogramm_Onlinefassung.pdf [26/10/2017].

Balzli, B. / Kamann, M., «Petry will den Begriff „völkisch“ positiv besetzen», Die Welt, 11 de septiembre de 2016. URL: https:/www.welt.de/politik/deutschland/article158049092/ Petry-will-den-Begriff-voelkisch-positiv-besetzen.html [13/10/2017]

Bausinger, H., «Volk und Sprache. Über eine mehrdeutige Beziehung», Zeitschrift für Volkskunde 87 (1991), 169-180.

Berbig, H. J., «Zur Terminologie von Volk, Nation und Reich in der neueren deutschen Geschichte», Zeitschrift für Religions- und Geistesgeschichte 28:1 (1976), 1-15. doi: 10.1163/157007376X00013

Bergem, W., Identitätsformationen in Deutschland. Wiesbaden: Verlag für Sozialwissenschaften 2005.

Berliner Zeitung, «Merkel zu Pegida-Rufen ,Das Volk ist jeder, der in diesem Land lebt“», 26.02.2017. URL: https://www.berliner-zeitung.de/politik/merkel-zu-pegida-rufen--dasvolk-ist-jeder--der-in-diesem-land-lebt--25820352 [ 26/10/2017].

Biermann, K., «,Völkisch“ ist nicht irgendein Adjektiv», Zeit Online 11 de septiembre de 2016. URL: https://www.zeit.de/kultur/2016-09/frauke-petry-afd-voelkisch-volk-begriff-geschichte [13/10/2017].

Brecht, B., «Fünf Schwierigkeiten beim Schreiben der Wahrheit», en: Brecht, B., Gesammelte Werke. Schriften zur Literatur und Kunst I. Vol. 18. Frankfurt am Main: Suhrkamp [1934] 1967, 222-239.

Chilton, P., «'The people’ in populist discourse. Using neuro-cognitive linguistics to understand political meanings», Journal of Language and Politics 16:4 (2017), 582-594. doi: 10.1075/jlp.17031.chi

Christlich Demokratische Union Deutschlands (CDU), Für ein Deutschland, in dem wir gut und gerne leben. Regierungsprogramm 2017-2021. URL: https://www.cdu.de/system/ tdf/media/dokumente/170703regierungsprogramm2017.pdf?file=1\&type=field_collection_item\&id=9932 [26/10/2017].

Connor, W., Ethnonationalism: the quest for understanding. Princeton: University Press 1994.

Conversi, D. (ed.), Ethnonationalism in the contemporary world: Walker Connor and the study of nationalism. Londres: Routledge 2002.

Die Grünen, Zukunft wird aus Mut gemacht. Bundestagswahlprogramm 2017. URL: https://cms.gruene.de/uploads/documents/BUENDNIS_90_DIE_GRUENEN_ Bundestagswahlprogramm_2017.pdf [26/10/2017].

Die Linke, Sozial. Gerecht. Frieden. Für Alle. Die Zukunft, für die wir kämpfen! DIE LINKE. Langfassung des Wahlprogramms zur Bundestagswahl 2017. URL: https://www. die-linke.de/fileadmin/download/wahlen2017/wahlprogramm2017/die_linke_wahlprogramm_2017.pdf [26/10/2017]. 
Digitales Wörterbuch der deutschen Sprache (DWDS). Ein Projekt der Berlin-Brandenburgischen Akademie der Wissenschaften. URL: https://dwds.de/ [26/10/2017].

Duden, Das Universalwörterbuch. 7. Auflage. Mannheim: Bibliographisches Institut. Versión digital en CD-ROM 2011.

Frankfurter Allgemeine Zeitung, «Petry sieht ,völkisch“ als positiven Begriff», 11 de septiembre de 2016. URL: http://www.faz.net/aktuell/politik/inland/afd-petry-sieht-voelkisch-als-positiven-begriff-14430103.html [13/10/2017].

Freie Demokraten (FDP), Denken wir neu. Das Programm der Freien Demokraten zur Bundestagswahl 2017: ,,Schauen wir nicht länger zu“. URL: https://www.fdp.de/sites/default/files/uploads/2017/08/07/20170807-wahlprogramm-wp-2017-v16.pdf [26/10/2017].

Freiheitliche Partei Österreichs (FPÖ), Österreicher verdienen Fairness. Freiheitliches Wahlprogramm zur Nationalratswahl 2017. URL: https://www.fpoe.at/fileadmin/user upload/www.fpoe.at/dokumente/2017/Wahlprogramm2017/Wahlprogramm_8_9_low. pdf [26/10/2017].

Gardt, A., «Nation und Sprache in der Zeit der Aufklärung», en: Gardt, A. (ed.), Nation und Sprache. Die Diskussion ihres Verhältnisses in Geschichte und Gegenwart. Berlín/Nueva York: de Gruyter 2000a, 169-198.

Gardt, A., «Sprachnationalismus zwischen 1850 und 1945», en: Gardt, A. (ed.), Nation und Sprache. Die Diskussion ihres Verhältnisses in Geschichte und Gegenwart. Berlín/Nueva York 2000b, 247-271.

Grimm, J. / W., Deutsches Wörterbuch. Leipzig: S. Hirzel, vol. 26 1854-1961.

Grünen, Das ist Grün. Wahlprogramm der Grünen. Nationalratswahl 2017. URL: https:// www.gruene.at/partei/programm/wahlprogramme [26/10/2017].

Handelsblatt, AfD-«Vizechefin beleidigt deutsche Nationalmannschaft», 8.07.2016. URL: https:/www.handelsblatt.com/politik/deutschland/beatrix-von-storch-der-islam-gehoertnicht-zu-deutschland/13848914-3.html [24/10/2017].

Hermanns, F., «,Volk' und ,Nation“. Zur Semantik zweier geschichtsmächtiger Begriffe», Der Deutschunterricht 2 (2003), 26-36.

Hildebrandt, L., Deutsch. Wiesbaden: Springer Fachmedien 1980.

Hoffmann, L., «Das ,Volk‘. Zur ideologischen Struktur eines unvermeidbaren Begriffs», Zeitschrift für Soziologie 20/3 (1991), 191-208.

Jakobs, H., «Diot und Sprache. Deutsch im Verband der Frankenreiche (8. bis frühes 11. Jahrhundert)», en: Gardt, A. (ed.), Nation und Sprache. Die Diskussion ihres Verhältnisses in Geschichte und Gegenwart. Berlín/Nueva York: de Gruyter 2000, 7-46.

Knape, J., «Humanismus, Reformation, deutsche Sprache und Nation», en: Gardt, A. (ed.), Nation und Sprache. Die Diskussion ihres Verhältnisses in Geschichte und Gegenwart. Berlín/Nueva York: de Gruyter 2000, 103-138.

Kopperschmidt, J., «Sollte man um Worte streiten? Historische und systematische Anmerkungen zur politischen Sprache», en: Liedtke, F. / Wengeler, M. / Böke, K. (eds.), Begriffe besetzen. Strategien des Sprachgebrauchs in der Politik. Opladen: Westdeutscher Verlag 1991, 70-89.

Koselleck, R., «Volk, Nation, Nationalismus, Masse», en: Brunner, O. / Conze, W. / Koselleck, R. (eds.), Geschichtliche Grundbegriffe. Historisches Lexikon zur politisch-sozialen Sprache in Deutschland. Vol. 7. Stuttgart: Klett-Cotta 1992, 141-431.

Lerchner, G., «Nation und Sprache im Spannungsfeld zwischen Sprachwissenschaft und Politik in der Bundesrepublik und der DDR bis 1989», en: Gardt, A. (ed.), Nation und Sprache. Die Diskussion ihres Verhältnisses in Geschichte und Gegenwart. Berlín/Nueva York: de Gruyter 2000, 273-302. 
Lötzsch, R., «Was ist ein Volk und was eine Nation?», UTOPIE kreativ 103/104 (1999), 15-30. Müller, F., Wer ist das Volk? Die Grundfrage der Demokratie. Berlín: Duncker \& Humblot 1997. NEOS, Zukunftsmanifest. URL: https://ichtuwas.neos.eu/manifest [26/10/2017].

Österreichische Volkspartei (ÖVP), Das Programm der Liste Sebastian Kurz - die neue Volkspartei zur Nationalratswahl 2017. Der neue Weg. URL: https://www.oevp.at/Programme-Statuten-Logos [26/10/2017].

Priester, K., «Wesensmerkmale des Populismus», Aus Politik und Zeitgeschichte (APuZ) 5-6 (2012). URL: http://www.bpb.de/apuz/75848/wesensmerkmale-des-populismus?p=all [23/10/2017].

Retterath, J., “Was ist das Volk?”. Volks- und Gemeinschaftskonzepte der politischen Mitte in Deutschland 1917-1924. Berlín: De Gruyter 2016.

Republik Österreich Bundesministerium für Inneres: Volksbefragung 2013. URL: http:// vb2013.bmi.gv.at [19/10/2017].

SketchEngine. URL: https://www.sketchengine.eu/ [26/10/2017].

Sozialdemokratische Partei Deutschlands (SPD), Zeit für mehr Gerechtigkeit. Unser Regierungsprogramm für Deutschland. URL: https://www.spd.de/fileadmin/Dokumente/ Regierungsprogramm/SPD_Regierungsprogramm_BTW_2017_A5_RZ_WEB.pdf [26/10/2017].

Sozialdemokratische Partei Österreichs (SPÖ), Das Programm für Wohlstand, Sicherheit \& gute Laune. Damit Sie bekommen, was Ihnen zusteht. Mehr Netto v. Brutto. Gleiche Chancen. Sichere Pensionen. URL: https://spoe.at/Plan-A-2017.pdf [26/10/2017].

Staatsgesetzblatt für die Republik Österreich 1. Mai 1945. URL: https://www.ris.bka.gv.at/ Dokumente/BgblPdf/1945_1_0/1945_1_0.pdf [11/01/2018].

Süddeutsche Zeitung, «Petry will Begriff, „völkisch“ positiv besetzen», 11 de septiembre de 2016. URL: http://www.sueddeutsche.de/politik/alternative-fuer-deutschland-petry-willbegriff-voelkisch-positiv-besetzen-1.3156403 [13/10/2017]

Thomas, H., «Sprache und Nation. Zur Geschichte des Wortes deutsch vom Ende des 11. bis zur Mitte des 15. Jahrhunderts», en: Gardt, A. (ed.), Nation und Sprache. Die Diskussion ihres Verhältnisses in Geschichte und Gegenwart. Berlín/Nueva York: de Gruyter 2000, 47-101.

Twitter Frauke Petry. URL: https://twitter.com/FraukePetry [26/10/2017].

von Polenz, P., Deutsche Sprachgeschichte. Vom Spätmittelalter bis zur Gegenwart. Vol. III. Berlín: de Gruyter 1999.

von Senger, H., «Literatur und Kunst - nicht nur Gegenstand der Ästhetik, sondern auch der ,Soziagogie‘ (Lehre von der Gesellschaftslenkung)?», en: Margagliotta, G. M. A., Robiglio, A. A. (eds.), Art, Intellect and Politics. A Diachronic Perspective. Leiden: Brill 2013, 23-67.

Wahrig-Burfeind, R., Brockhaus Wahrig Deutsches Wörterbuch. 9., vollständig neu bearbeitete und aktualisierte Auflage. Gütersloh/Múnich: Brockhaus. Versión digital 2011.

Wiktorowicz, J., «Die Bedeutungsveränderungen der Personalkollektiva Volk und Nation», en: Wiesinger, P. (ed.), Akten des X. Internationalen Kongresses Wien 2000. Zeitenwende - Die Germanistik auf dem Weg vom 20. ins 21. Jahrhundert. Vol. II. Berna: Peter Lang 2002, 343-350.

Wildt, M., Volk, Volksgemeinschaft, AfD. Hamburgo: Hamburger Edition. 2017. 